\title{
Rashba Spin-Orbit Coupling in Image Potential States
}

\author{
S. Tognolini, ${ }^{1}$ S. Achilli, ${ }^{2}$ L. Longetti ${ }^{3}$ E. Fava, ${ }^{1}$ C. Mariani, ${ }^{3}$ M. I. Trioni, ${ }^{2}$ and S. Pagliara ${ }^{1}$ \\ ${ }^{1}$ I-LAMP and Dipartimento di Matematica e Fisica, Università Cattolica, 25121 Brescia, Italy \\ ${ }^{2}$ Dipartimento di Chimica, Università degli Studi di Milano and CNR-ISTM, via Golgi 19, 20133 Milano, Italy \\ ${ }^{3}$ Dipartimento di Fisica, CNISM, Università di Roma "La Sapienza", 00185 Roma, Italy \\ (Received 22 October 2014; revised manuscript received 24 March 2015; published 22 July 2015)
}

\begin{abstract}
The search in two-dimensional condensed matter systems of Rashba-type spin-polarized electronic states is aimed by the possibility to control and manipulate the spin orientation. In this Letter, for the first time, we report on the experimental evidence of a Rashba-type spin splitting in the $n=1$ image potential state. The image potential state Rashba splitting here measured at the graphene/Ir(111) interface, as confirmed by theoretical considerations, can be detectable to any metal surface with a significant spin-orbit coupling.
\end{abstract}

DOI: 10.1103/PhysRevLett.115.046801

PACS numbers: 73.20.-r, 71.70.Ej, 73.22.Pr

The Rashba effect, extensively studied in these last years for the potential impact on spintronics and magnetoelectrics, is one of the most important consequences of spinorbit interaction. The standard model for the Rashba effect relies on an isotropic two-dimensional electron gas (2DEG) that under broken inversion symmetry along the direction perpendicular to the 2DEG plane splits its spin-degenerate parabolic band into oppositely spin-polarized subbands displaced in opposite directions in momentum space [1-3].

Spin-polarized electronic states have been measured in a semiconductor heterostructure where a two-dimensional electron gas (2DEG) is confined in the band-bending region that behaves as a potential gradient perpendicular to the interface plane [4]. A much larger Rashba effect is known to exist at several heavy metal surfaces where the $2 \mathrm{DEG}$ is replaced by surface states and the potential gradient is given by the surface potential barrier. In this context, the surface states (SS) at the $L$ gap of $\mathrm{Au}(111)$ represents the prototype of a Rashba-split states [5,6], even if a giant Rashba effect has been measured also on the $\operatorname{Ir}(111)$ surface state [7].

In addition to the occupied surface states, all these (111) surfaces support unoccupied image potential states (IPS) that are bound by the image potential resulting from the polarization charge induced by an electron in front of a metal surface [8].

To date, although it is in principle possible and it has been theoretically predicted [9], no experimental evidence of the IPS Rashba splitting has been reported in the literature. Indeed, the discovery of a Rashba spin-orbit coupling on IPS could be exploited in the photoinduced charge transfer processes at the interface to inject spinpolarized electrons. IPS, in fact, thanks to delocalization of the associated wave function in the plane parallel to the metal surface together with the large spatial extension into the vacuum, represents an efficient photoinduced charge transfer channel at the adsorbate-metal interface [10-12]. The possibility to inject spin-polarized electrons through the image potential state paves new ways in the field of the femtosecond switching of magnetism [13,14].

In this Letter, by using circularly polarized femtosecond laser pulses in nonlinear photoemission measurements, we show for the first time that the $n=1$ IPS exhibits a Rashba effect. The feasibility to detect such a small Rashba splitting has been possible thanks to the very high resolution of the measurements. The Rashba splitting measured on the $n=1$ IPS, is 45 times smaller than that measured on the occupied SS of the same surface.

To achieve and better highlight the Rashba effect in the image potential state we have chosen the graphene/Ir(111) interface. Graphene grows as an almost free-standing ordered sheet on this surface [15-18] thanks to the weak interaction between graphene and $\operatorname{Ir}(111)$. As a consequence, the giant Rashba effect of the surface state measured on the $\operatorname{Ir}(111)$ surface remains unaffected [7]. Epitaxial graphene decreases the large work function of the $\operatorname{Ir}(111)$ allowing us to efficiently populate, by using photon energies in the near ultraviolet region, the IPS also for $k_{\|} \neq 0$ where the Rashba splitting is measurable. Moreover, it has been demonstrated that graphene on $\operatorname{Ir}(111)$ acts as a buffer layer capable of decoupling adsorbed molecules from the metallic substrate [16,19-21], leaving undisturbed a possible spin-polarized electron transfer from the metal to the adsorbed molecule assisted by image potential state.

Details on the single-layer graphene grown on $\operatorname{Ir}(111)$ and on the nonlinear photoemission experimental setup are reported in the Supplemental Material [22]. The giant Rashba effect on SS of the $\operatorname{Ir}(111)$ covered with a single graphene layer is well measurable by collecting the angular dispersion of the photoemission spectra (Fig. 1). To discriminate the occupied from the unoccupied surface states, the spectra have been acquired with two photon energies, the first larger $[h \nu=6.24 \mathrm{eV}$, Fig. 1(b)] and the second smaller $[h \nu=3.12 \mathrm{eV}$, Fig. 1(a)] than the sample work function $(\Phi=4.45 \pm 0.05 \mathrm{eV})$. The occupied 
(a) $h v=3.12 \mathrm{eV}$
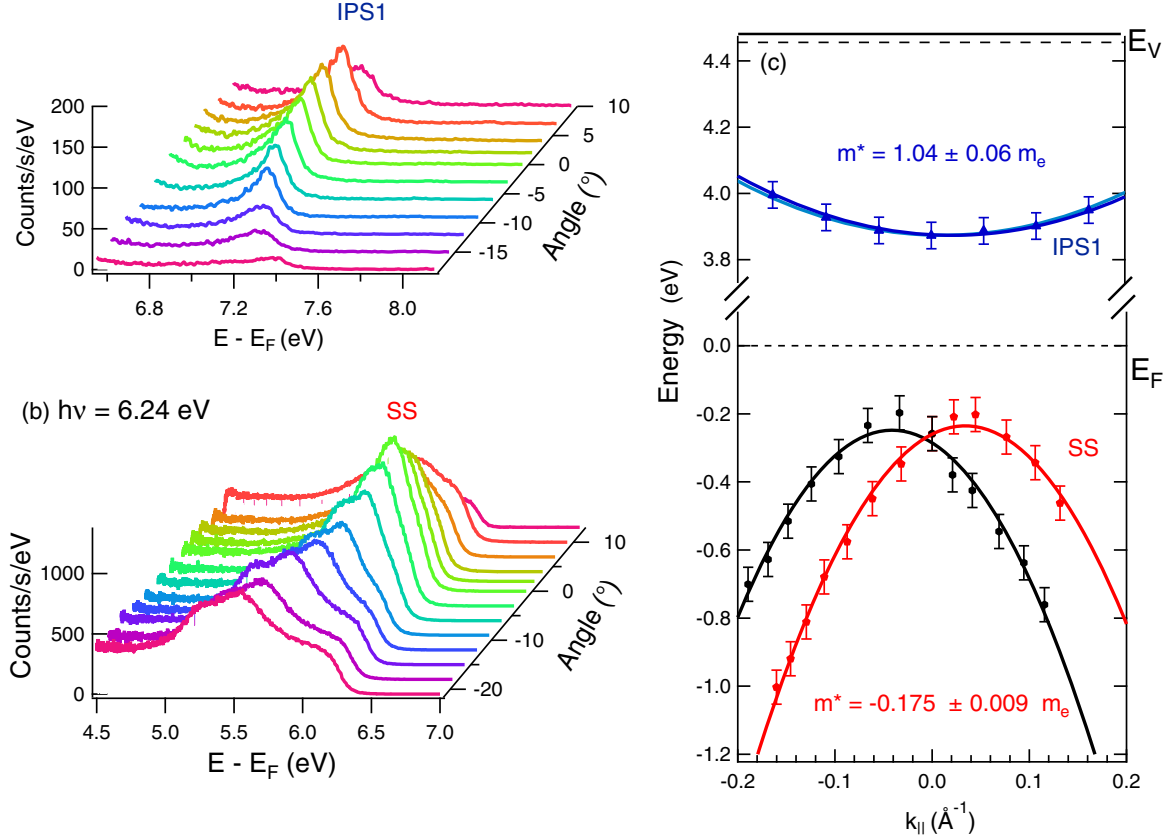

FIG. 1 (color online). (a) Angle-resolved nonlinear photoemission spectra collected with a photon energy of $3.12 \mathrm{eV}$ and $P$-polarized light at the graphene/Ir(111) interface. (b) Angle-resolved linear photoemission spectra collected with a photon energy of $6.24 \mathrm{eV}$ and $P$-polarized light at the graphene/Ir(111) interface. (c) Energy position versus $k_{\|}$momentum for the first image potential state IPS1 and the surface states SS. The parabolic fit (line) of the data gives the effective mass and the Rashba effect reported in the figure.

Rashba-type $\mathrm{SS}$ is measurable at $E-E_{F} \sim 6 \mathrm{eV}$ [Fig. 1(b)], while the feature at $E-E_{F} \sim 7 \mathrm{eV}$ [Fig. 1(a)] is due to the first unoccupied image potential state (IPS1). The resulting binding energy at the $\bar{\Gamma}$ point is $0.58 \pm 0.02 \mathrm{eV}$ for IPS1 with respect to the vacuum level and $0.26 \pm 0.05 \mathrm{eV}$ for the SS $(0.195 \pm 0.05 \mathrm{eV}$ considering the minimum one, out of the $\bar{\Gamma}$ point), with respect to the Fermi level. In agreement with Refs. [7,28] the SS binding energy results shifted of about $150 \mathrm{meV}$ with respect to the pristine $\operatorname{Ir}(111)$ surface state.

The $E\left(k_{\|}\right)$dispersion of SS and IPS1 sampled by the spectra angular dispersion is displayed in Fig. 1(c). The IPS1 $k_{\|}$dispersion fits well with a parabolic function with an effective mass close to the free-electron mass, $m^{*}=1.04 \pm 0.06 m_{\mathrm{e}}$. The occupied surface state appears as two identical parabolic downward-dispersing structures resembling the dispersion of a Rashba-type spin-split surface state [1]. The two $E_{ \pm}\left(k_{\|}\right)$parabolas result well interpolated by $[7,28-30]$

$$
E_{ \pm}\left(k_{\|}\right)=E_{0}+\frac{\hbar^{2} k_{\|}^{2}}{2 m^{*}} \pm \alpha_{R}\left|k_{\|}\right|
$$

where the two parabolas are shifted relative to the $k_{\|}=0$ ( $\bar{\Gamma}$ point) by $\Delta k_{\|}=\left(m^{*} \alpha_{R}\right) / \hbar^{2}$. The splitting relative to the $\bar{\Gamma}$ point amounts to $\Delta k_{\|}=0.0377 \pm 0.0026 \AA^{-1}$, resulting in the giant Rashba effect with $\alpha_{R}=(1.64 \pm 0.18) \times$ $10^{-10} \mathrm{eV}$ in agreement with the values reported in the literature [7]. Accordingly to the weak interaction between graphene and $\operatorname{Ir}(111)$, the presence of graphene does not influence either the dispersion of the surface state or the Rashba splitting, preserving the giant size of the $\operatorname{Ir}(111)$ surface state splitting.

When the Rashba effect is sufficiently large as for the $\operatorname{Ir}(111) \mathrm{SS}$, the spin-orbit splitting $\Delta E_{\mathrm{SO}}$ is easily detectable as soon as we move away from the $\bar{\Gamma}$ point. Conversely, as recently reported for the surface states of topological insulators [31-33], when the Rashba effect is not so large, circularly polarized laser pulses or a spin resolved detector are necessary. For this reason, in order to find a trace of a possible Rashba effect on the IPS1, we have irradiated the graphene/Ir(111) interface with circularly polarized laser pulses at $h \nu=4.64 \mathrm{eV}$. This pump photon energy has been suitably chosen to better highlight this effect. Being the SS-IPS1 energy difference at the $\bar{\Gamma}$ point equal to $4.1 \mathrm{eV}$, the $h \nu=4.64 \mathrm{eV}$ photon energy allows us to populate, absorbing one-photon, IPS1 in a quasiresonant way from SS at $k_{\|} \neq 0$. Since the work function of graphene/Ir(111) interface $(\Phi=4.45 \pm 0.05 \mathrm{eV})$ is smaller than the chosen $h \nu=4.64 \mathrm{eV}$ photon energy, a potential difference of $\Delta V=+0.7 \mathrm{~V}$ has been applied to the sample to remove the linear photoemission contribution (1PPE of Fig. 2) in the spectrum. The two structures present in the two-photon contribution of the photoemission spectrum collected at $k_{\|}=0$ in $P$ polarization (Fig. 2) represent the IPS1 and the $n=2$ image state (IPS2), as also confirmed by the $k_{\|}$ 


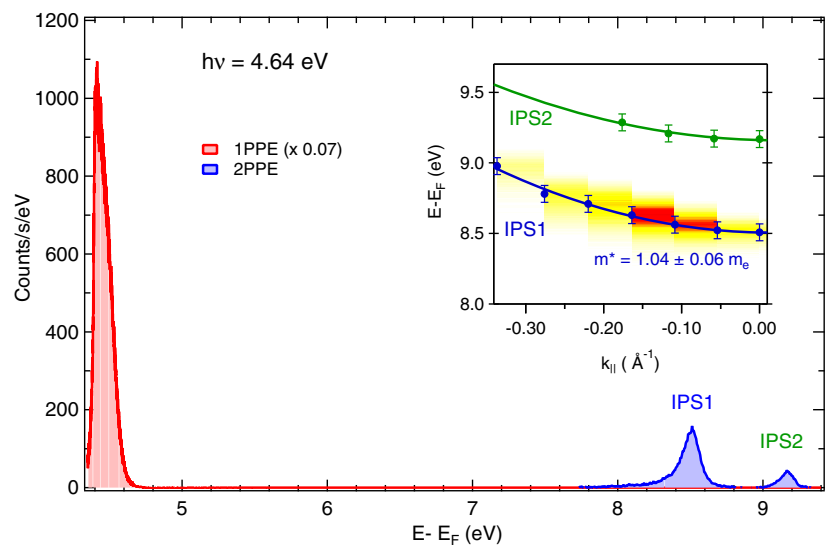

FIG. 2 (color online). Comparison between nonlinear photoemission (blue) and linear photoemission (red) spectra collected at a photon energy of $4.64 \mathrm{eV}$ at $k_{\|}=0$ and $P$-polarized light. Being that the work function $(\Phi=4.45 \pm 0.05 \mathrm{eV})$ is smaller than the laser photon energy, in order to measure the nonlinear contribution in the photoemission spectrum, a positive potential of $+0.7 \mathrm{~V}$ was applied to the sample. In the inset we report the angular dispersion of the photoemission spectra collected at a photon energy of $4.64 \mathrm{eV}$.

dispersion collected in these experimental conditions (inset of Fig. 2).

Figure 3 reports the left- and right-hand circularly polarized two-photon photoemitted IPS1 structures, collected at emission angles corresponding to the electron wave vectors $k_{\|}= \pm 0.16 \AA^{-1}$ and $k_{\|}=0$. While the IPS1 features at $k_{\|}=0$ with left and right circular polarization well overlap, a dichroic signal is undoubtedly visible at $k_{\|} \neq 0$.

The dichroic signal has been verified by using two different photon energies. The $h \nu=4.64 \mathrm{eV}$ is able to populate in a quasiresonant way the IPS1 from the SS in particular at $k_{\|} \neq 0$. At this photon energy the IPS1 signal is more intense; however, the population mechanism from the SS could affect the dichroism measured on the IPS1. At $h \nu=3.12 \mathrm{eV}$ (inset of Fig. 3), the IPS1 is a third order process being the multiphotonic order $\mathrm{MPO}=3$ (see Supplemental Material [22]). It is populated by absorbing two photons and then from states deeper in energy from the Fermi level than the SS. The comparable dichroic signal, measured with the two photon energies, nullifies the doubt that initial state effects can be at the origin of the dichroism observed on IPS1.

To better highlight it, we calculated the difference between the best fits of the IPS1 features collected with the two circular polarizations: the estimated spin orbit splitting results $\Delta E_{\mathrm{SO}}=11.5 \pm 2.0 \mathrm{meV}$ at $k_{\|}=$ $\pm 0.16 \AA^{-1}$. Comparing this value with the spin-orbit splitting measured on the $\mathrm{SS}\left(\Delta E_{\mathrm{SO}}=525 \mathrm{meV}\right)$ at the same $k_{\|}$value, we find that on the IPS1 the effect is fortyfive times smaller than on the SS. Estimating a $k_{\|}$splitting relative to the $\bar{\Gamma}$ point of $\Delta k_{\|}=0.005 \pm 0.001 \AA^{-1}$, the
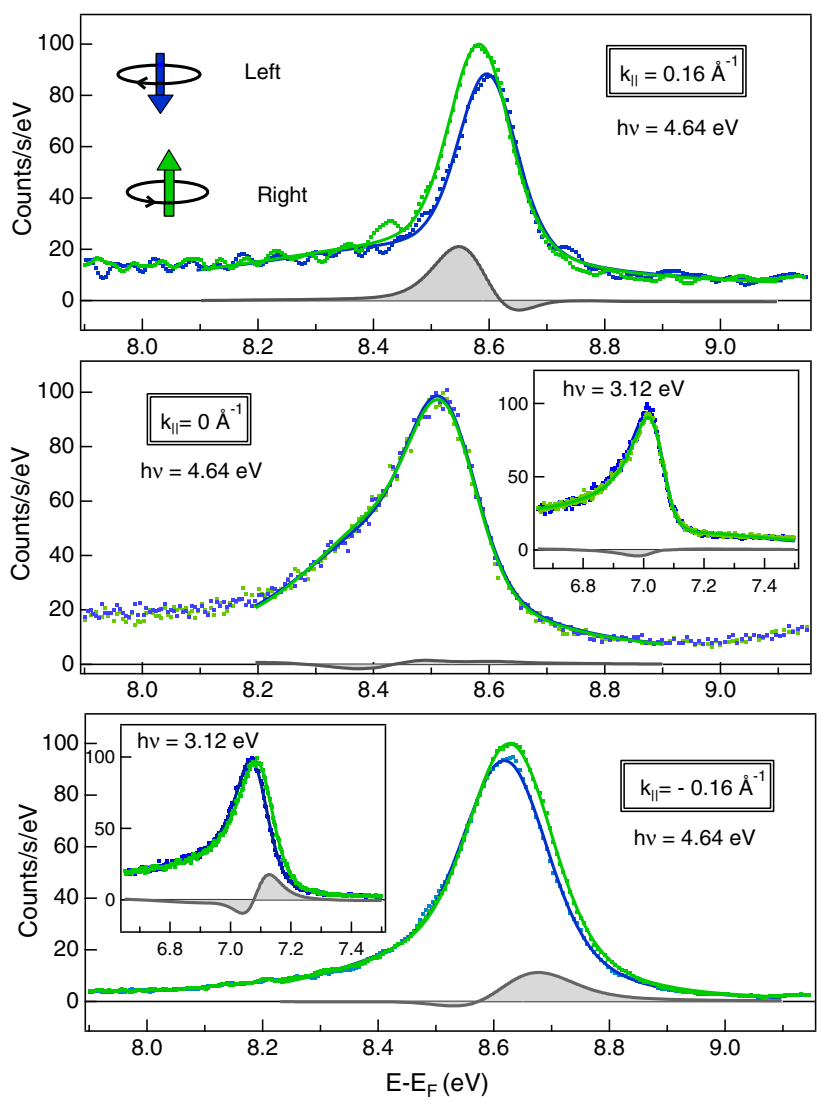

FIG. 3 (color online). Comparison between nonlinear photoemission spectra collected at a photon energy of $4.64 \mathrm{eV}$ in right (green) and left (blue) circular polarization, and the difference between the best fit of the corresponding signals (gray). The measurements are acquired at the $\bar{\Gamma}$ point $\left(\theta=0^{\circ}\right)$ and at symmetric angles $\left(\theta=9^{\circ}\right.$ and $\left.\theta=-9^{\circ}\right)$ corresponding to $k_{\|}=0$ and $k_{\|}= \pm 0.16 \AA^{-1}$, respectively. In the inset, nonlinear photoemission spectra collected at a different photon energy $3.12 \mathrm{eV}$ by changing the circular polarization from right (green) to left (blue) at the same $k_{\|}$values $\left(k_{\|}=0\right.$ and $k_{\|}=-0.16 \AA^{-1}$, the data at $k_{\|}=+0.16 \AA^{-1}$ are not available for experimental constraint). The dichroic signals measured with the two photon energies are comparable.

Rashba effect for the IPS1 results to be $\alpha_{R}=$ $(3.6 \pm 0.6) \times 10^{-12} \mathrm{eV} \mathrm{m}$.

We note that, while the energy shift of the IPS1 is comparable, a difference exists in the intensity of the dichroic signal measured with $h \nu=4.64 \mathrm{eV}$ and $h \nu=3.12 \mathrm{eV}$. We believe that this effect can be ascribed to the population mechanism.

The different Rashba splitting of SS and IPS1 can be explained by their different spatial localization and decay at the surface, making them differently affected by the SO coupling.

In particular, this has been invoked to explain the larger SO splitting observed for SS of $\mathrm{Au}(111)$ with respect to $\operatorname{Ag}(111)$ [34], and the enhancement and reduction of the Rashba splitting in the surface state of $\mathrm{Bi} / \mathrm{Cu}(111)$ upon 
$\mathrm{Na}$ and $\mathrm{Xe}$ adsorption, respectively [35]. In Ref. [9] McLaughlan et al., using a relativistic multiple scattering theory, calculated the Rashba splitting of image state for Pt, Ir, and Au surfaces finding that, for $n=1$, it is 1 order of magnitude smaller with respect to $\mathrm{Au}(111) \mathrm{SS}$, due to the smaller penetration of the wave function into the surface. In particular, for the first image state of the clean $\operatorname{Ir}(111)$ surface they found $\alpha_{R}=2.8 \times 10^{-12} \mathrm{eV} \mathrm{m}$.

It is, in principle, not obvious that a similar splitting could be found also in the presence of graphene. Indeed the graphene sheet is expected to perform a screening effect on external image charges, leading to a different spatial localization of the wave function at the surface. In order to investigate this aspect we have performed $a b$ initio calculations of the electronic properties of graphene/ $\operatorname{Ir}(111)$. Details about the calculation are furnished in the Supplemental Material [22].

In Fig. 4, we report the squared modulus of the first image state wave function along $z$ for clean $\operatorname{Ir}(111)$ (red line) and graphene/Ir(111) (blue line), which is the relevant quantity to estimate the Rashba parameter. The latter can be indeed expressed as

$$
\alpha_{R}=2 / c^{2} \int|\psi(z)|^{2} \partial_{z} V d z
$$

where $|\psi(z)|^{2}$ is the charge distribution of the surface state along the surface normal and $\partial_{z} V$ is the derivative of the atomic potential $[35,36]$. Because of the very short range nature of the last term, the integral in Eq. (2) has to be determined in a very small region around the atomic core. Being the gradient of the atomic potential antisymmetric in the relevant region around the nuclear position, only the asymmetric part of the surface state charge density along $z$, which is related to the asymmetry of the surface potential, contributes to the integral. In particular, the Rashba parameter will depend on the amplitude of the wave function at the surface and on its decay into the substrate.

From the results reported in Fig. 4 we find that in the presence of graphene, the amplitude of IPS1 charge distribution on the first Ir layer is only 3.5 times smaller than that on clean $\operatorname{Ir}(111)$ while the decay factor is almost unchanged. Hence a small Rashba splitting is expected for this state. The linear screening density, due to an external charge [37] (see the inset in Fig. 4), confirms the only partial screening of the carbon layer. In the presence of graphene, the image plane does not follow rigidly the surface layer: its outward shift of $2.72 \AA$ with respect to the clean surface is indeed smaller than the Ir-graphene distance, equal to $3.5 \AA$.

The comparison with the charge density distribution of the SS of $\operatorname{Ir}(111)$ (green line), characterized by amplitude 2 order of magnitude larger than IPS1 and a more marked asymmetry in the surface layer, confirms a more sizable

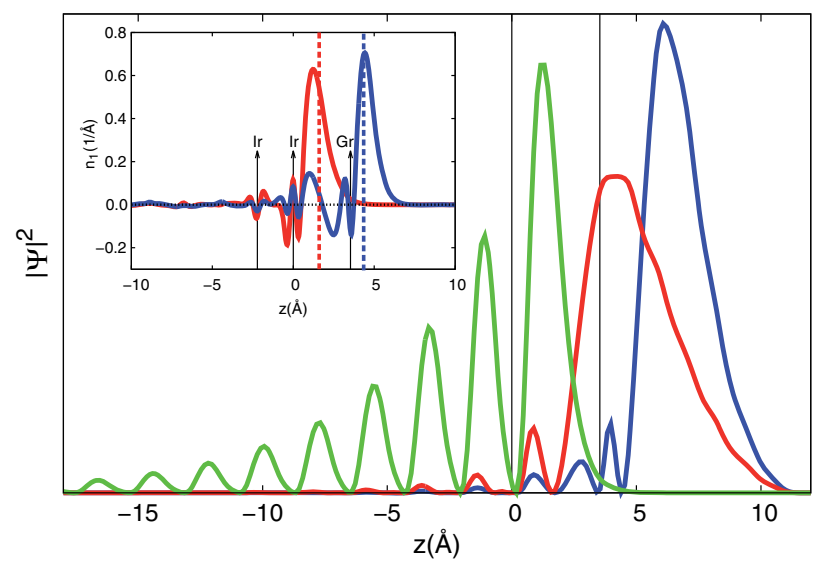

FIG. 4 (color online). Squared amplitude of the wave functions at $\bar{\Gamma}$ of SS of $\operatorname{Ir}(111)$ (green), IPS1 of $\operatorname{Ir}(111)$ (red), and IPS1 of graphene/Ir(111) (blue). Vertical lines correspond to the Ir surface layer $(z=0)$ and graphene plane. In the inset: plot of the linear screening density $n_{1}$ of $\operatorname{Ir}(111)$ and graphene/ $\operatorname{Ir}(111)$ due to the application of an external charge. The arrows mark nuclear positions while vertical dashed lines represent the image plane of the two systems.

Rashba splitting for this surface state, in agreement with the experimental measurements and the literature [9].

In conclusion, the Rashba effect has been experimentally revealed for the first time on the $n=1$ IPS. The value of the Rashba effect on the IPS1 results 45 times smaller than one measured on the same surface on the occupied SS. If the spin-orbit coupling is the same for both states, this difference can be ascribed both to the smaller amplitude of the IPS and to the smaller asymmetry of the image state charge density with respect to the SS around the Ir nuclear position. The presence of the graphene sheet is expected to reduce slightly this effect with respect to the clean surface case. This discovery, by exploiting the spin-split image state, could open new scenarios for the understanding and the control of the magnetism at interfaces.

Experimental assistance from Simone Lisi is deeply appreciated. C. M. thanks the PIK project EX-PRO-REL of Elettra Sincrotrone Trieste, the PRIN Grant No. 20105ZZTSE "GRAF" of the Italian Ministery for Research (MIUR) and Roma "La Sapienza" University funds. Moreover, S. P. thanks the MIUR for supporting this work under Contract No. PRIN 2010BNZ3F2 and acknowledges partial support from D.2.2 grants of the Università Cattolica.

[1] E. I. Rashba, Fiz. Tverd. Tela 2, 1224 (1960) [Sov. Phys. Solid State 2, 1109 (1960)].

[2] G. Dresselhaus, Phys. Rev. 100, 580 (1955).

[3] M. Heide, G. Bihlmayer, Ph. Mavropoulos, A. Bringer, and S. Blügel, $\Psi_{k}$ Newsletter 78, 1 (2006), http://www.psi-k.org/ newsletters/News_78/Highlight_78.pdf. 
[4] R. Winkler, Spin-Orbit Coupling Effects in Two-Dimensional Electron and Hole Systems, Springer Tracts in Modern Physics (Springer, Berlin, 2003), Vol. 191.

[5] S. LaShell, B. A. McDougall, and E. Jensen, Phys. Rev. Lett. 77, 3419 (1996).

[6] M. Hoesch, M. Muntwiler, V. N. Petrov, M. Hengsberger, L. Patthey, M. Shi, M. Falub, T. Greber, and J. Osterwalder, Phys. Rev. B 69, 241401(R) (2004).

[7] A. Varykhalov, D. Marchenko, M. R. Scholz, E. D. L. Rienks, T. K. Kim, G. Bihlmayer, J. Sánchez-Barriga, and O. Rader, Phys. Rev. Lett. 108, 066804 (2012).

[8] S. Hüfner, Photoelectron Spectroscopy, Advanced Texts in Physics (Springer, Berlin, 2003).

[9] J. R. McLaughlan, E. M. Llewellyn-Samuel, and S. Crampin, J. Phys. Condens. Matter 16, 6841 (2004).

[10] J. Güdde, W. Berthold, and U. Höfer, Chem. Rev. 106, 4261 (2006).

[11] E. V. Chulkov, V. M. Silkin, and P. M. Echenique, Surf. Sci. 437, 330 (1999).

[12] C. D. Lindstrom and X.-Y. Zhu, Chem. Rev. 106, 4281 (2006).

[13] J.-Y. Bigot, M. Vomir, and E. Beaurepaire, Nat. Phys. 5, 515 (2009).

[14] T. Li, A. Patz, L. Mouchliadis, J. Yan, T. A. Lograsso, I. E. Perakis, and J. Wang, Nature (London) 496, 69 (2013).

[15] I. Pletikosić, M. Kralj, P. Pervan, R. Brako, J. Coraux, A. T. N'Diaye, C. Busse, and T. Michely, Phys. Rev. Lett. 102, 056808 (2009).

[16] L. Massimi, S. Lisi, D. Pacilè, C. Mariani, and M. G. Betti, Beilstein J. Nanotechnol. 5, 308 (2014).

[17] A. B. Preobrajenski, M. L. Ng, A. S. Vinogradov, and N. Mårtensson, Phys. Rev. B 78, 073401 (2008).

[18] A. T. N'Diaye, J. Coraux, T. N. Plasa, C. Busse, and T. Michely, New J. Phys. 10, 043033 (2008).

[19] M. Scardamaglia, G. Forte, S. Lizzit, A. Baraldi, P. Lacovig, R. Larciprete, C. Mariani, and M. G. Betti, J. Nanopart. Res. 13, 6013 (2011).

[20] M. Scardamaglia, S. Lisi, S. Lizzit, A. Baraldi, R. Larciprete, C. Mariani, and M. G. Betti, J. Phys. Chem. C 117, 3019 (2013).

[21] M. Scardamaglia, C. Struzzi, S. Lizzit, M. Dalmiglio, P. Lacovig, A. Baraldi, C. Mariani, and M. G. Betti, Langmuir 29, 10440 (2013).
[22] See Supplemental Material at http://link.aps.org/ supplemental/10.1103/PhysRevLett.115.046801 for a detailed description of the experimental setup and nonlinear photoemission results, along with a description of the $a b$ initio calculations of the electronic properties of graphene/Ir(111). The Supplemental Material includes Refs. [23-27].

[23] T. Ohta, A. Bostwick, J. L. McChesney, T. Seyller, K. Horn, and E. Rotenberg, Phys. Rev. Lett. 98, 206802 (2007).

[24] D. Niesner, Ph.D. thesis, Naturwissenschaftlichen Fakultät der Friedrich-Alexander-Universität Erlangen-Nürnberg, 2013.

[25] P. Hohenberg and W. Kohn, Phys. Rev. 136, B864 (1964).

[26] J. M. Soler, E. Artacho, J. D. Gale, A. García, J. Junquera, P. Ordejón, and D. Sánchez-Portal, J. Phys. Condens. Matter 14, 2745 (2002).

[27] J. P. Perdew, K. Burke, and M. Ernzerhof, Phys. Rev. Lett. 77, 3865 (1996).

[28] J. Sánchez-Barriga, G. Bihlmayer, D. Wortmann, D. Marchenko, O. Rader, and A. Varykhalov, New J. Phys. 15, 115009 (2013).

[29] D. Niesner, Th. Fauster, J. I. Dadap, N. Zaki, K. R. Knox, P.-C. Yeh, R. Bhandari, R. M. Osgood, M. Petrović, and M. Kralj, Phys. Rev. B 85, 081402(R) (2012).

[30] D. Nobis, M. Potenz, D. Niesner, and Th. Fauster, Phys. Rev. B 88, 195435 (2013).

[31] M. R. Scholz, J. Sánchez-Barriga, J. Braun, D. Marchenko, A. Varykhalov, M. Lindroos, Y. J. Wang, H. Lin, A. Bansil, J. Minár et al., Phys. Rev. Lett. 110, 216801 (2013).

[32] M. R. Scholz, J. Sánchez-Barriga, D. Marchenko, A. Varykhalov, A. Volykhov, L. V. Yashina, and O. Rader, arXiv:1108.1053v2.

[33] C. Cacho, A. Crepaldi, M. Battiato, M. Cilento, F. Zacchigna, M. C. Richter, O. Heckmann, E. Springate, Y. Liu, S. S. Dhesi et al., Phys. Rev. Lett. 114, 097401 (2015).

[34] G. Bihlmayer, Y. M. Koroteev, P. M. Echenique, E. V. Chulkov, and S. Blügel, Surf. Sci. 600, 3888 (2006).

[35] H. Bentmann and F. Reinert, New J. Phys. 15, 115011 (2013).

[36] M. Nagano, A. Kodama, T. Shishidou, and T. Oguchi, J. Phys. Condens. Matter 21, 064239 (2009).

[37] S. Achilli, S. Caravati, and M. I. Trioni, Surf. Sci. 601, 4048 (2007). 